Kragujevac Journal of Mathematics

Volume 40(1) (2016), Pages 35-45.

\title{
ON THE CHARACTERIZATION OF A CLASS OF FOUR DIMENSIONAL MATRICES AND STEINHAUS TYPE THEOREMS
}

\author{
M. YEŞILKAYAGIL ${ }^{1}$ AND F. BAŞAR ${ }^{2}$
}

\begin{abstract}
In the present paper, we give the definitions of four dimensional conull and coregular matrices, and characterize the classes $\left(\mathcal{M}_{u}: \mathcal{C}_{\vartheta}\right)$, where $\vartheta \in\{p, p 0, f\}$ and $\mathcal{C}_{f}$ denotes the space of all almost convergent double sequences. Additionally, we give two Steinhaus type theorems related to the coercive/almost coercive four dimensional sequence to sequence matrix transformations.
\end{abstract}

\section{INTRODUCTION}

Referring Eizen and Laush [1] and Başar [2], we characterize the classes $\left(\mathcal{M}_{u}: \mathcal{C}_{\vartheta}\right)$, where $\vartheta \in\{p, p 0, f\}$. We quote some lemmas and also, we define the concept of conullity and coregularity of a four dimensional matrix. Additionally, as a new development of Steinhaus type theorems we prove that there is no matrix belonging to both of the classes $\left(\mathcal{M}_{u}: \mathcal{C}_{\vartheta}\right)$ and $\left(\mathcal{C}_{\vartheta}: \mathcal{C}_{\vartheta} ; P\right)$ with $\vartheta \in\{b p, f\}$ of four dimensional matrices.

\section{Definitions, Notations and Background}

We denote the set of all complex valued double sequences by $\Omega$ which is a vector space with coordinate-wise addition and scalar multiplication. Any vector subspace of $\Omega$ is called as a double sequence space. A double sequence $x=\left(x_{m n}\right)$ of complex numbers is said to be bounded if $\|x\|_{\infty}=\sup _{m, n \in \mathbb{N}}\left|x_{m n}\right|<\infty$, where $\mathbb{N}=\{0,1,2, \ldots\}$. It is known that the space $\mathcal{M}_{u}$ of all bounded double sequences is a Banach space with the norm $\|\cdot\|_{\infty}$. If there is an $l \in \mathbb{C}$ such that for every $\varepsilon>0$ there exists $n_{0}=n_{0}(\varepsilon) \in \mathbb{N} \ni\left|x_{m n}-l\right|<\varepsilon$ for all $m, n>n_{0}$, then we call that the double

Key words and phrases. Double sequence, double series, Pringsheim convergence, Steinhaus type theorems, matrix transformations.

2010 Mathematics Subject Classification. 40C05.

Received: June 2, 2015.

Accepted: December 13, 2015. 
sequence $x=\left(x_{m n}\right) \in \Omega$ is convergent in the Pringsheim's sense to the limit $l$ and write $p-\lim _{m, n \rightarrow \infty} x_{m n}=l$, [3]; where $\mathbb{C}$ denotes the complex field. By $\mathcal{C}_{p}$, we denote the space of all convergent double sequences in the Pringsheim's sense. It is well-known that there are such sequences in the space $\mathcal{C}_{p}$ but not in the space $\mathcal{M}_{u}$. Indeed, if we define the sequence $x=\left(x_{k l}\right)$ by

$$
x_{k l}:= \begin{cases}k, & k \in \mathbb{N}, l=0, \\ l, & l \in \mathbb{N}, k=0 \\ 0, & k, l \in \mathbb{N} \backslash\{0\}\end{cases}
$$

for all $k, l \in \mathbb{N}$, then it is trivial that $x \in \mathcal{C}_{p} \backslash \mathcal{M}_{u}$, since $p-\lim _{k, l \rightarrow \infty} x_{k l}=0$ but $\|x\|_{\infty}=\infty$. So, we can consider the space $\mathcal{C}_{b p}$ of the double sequences which are both convergent in the Pringsheim's sense and bounded, i.e., $\mathfrak{C}_{b p}=\mathcal{C}_{p} \cap \mathcal{M}_{u}$. A sequence in the space $\mathcal{C}_{p}$ is said to be regularly convergent if it is a single convergent sequence with respect to each index and denote the space of all such sequences by $\mathcal{C}_{r} . \mathrm{By}_{p 0}$, we denote the space of all double sequences converging to 0 in the Pringsheim's sense. Also by $\mathcal{C}_{b p 0}$ and $\mathcal{C}_{r 0}$, we denote the spaces of all double null sequences contained in the sequence spaces $\mathcal{C}_{b p}$ and $\mathcal{C}_{r}$, respectively. Móricz [4] proved that $\mathcal{C}_{b p}, \mathcal{C}_{b p 0}, \mathcal{C}_{r}$ and $\mathcal{C}_{r 0}$ are Banach spaces with the norm $\|\cdot\|_{\infty}$.

The concept of almost convergence for single sequences was introduced by Lorentz [6] and for double sequences by Móricz and Rhoades [5]. A double sequence $x=\left(x_{m n}\right)$ of complex numbers is said to be almost convergent to a generalized limit $L$ if

$$
p-\lim _{q, r \rightarrow \infty}\left|\frac{1}{(q+1)(r+1)} \sum_{k=m}^{m+q} \sum_{l=n}^{n+r} x_{k l}-L\right|=0 \text { uniformly in } m, n .
$$

In this case, $L$ is called the $f_{2}$-limit of $x$. We denote the space of all almost convergent double sequences by $\mathcal{C}_{f}$.

If we define the sequence $x=\left(x_{k l}\right)$ with respect to the natural numbers $q$ and $r$ by

$$
x_{k l}:=\left\{\begin{array}{cc}
k l, & 0 \leq k \leq q, 0 \leq l \leq r, \\
0, & \text { otherwise }
\end{array}\right.
$$

for all $k, l \in \mathbb{N}$, one can easily see that $x \in \mathcal{C}_{p}$. But, since the limit

$$
p-\lim _{q, r \rightarrow \infty} \frac{1}{(q+1)(r+1)} \sum_{k=m}^{m+q} \sum_{l=n}^{n+r} x_{k l}=p-\lim _{q, r \rightarrow \infty}\left(m+\frac{q}{2}\right)\left(n+\frac{r}{2}\right)
$$

does not exist for each $m$ and $n, x \notin \mathcal{C}_{f}$. So, we note that a convergent double sequence in the Pringsheim's sense need not be almost convergent. However every bounded convergent double sequence is almost convergent and every almost convergent double sequence is bounded. Four dimensional matrices mapping every almost convergent double sequence into a $b p$-convergent double sequence with the same limit are said to be strongly regular and considered by Móricz and Rhoades [5].

Let $\lambda$ be a space of double sequences converging with respect to some linear convergence rule $\vartheta-\lim : \lambda \rightarrow \mathbb{C}$. The sum of a double series $\sum_{k, l} x_{k l}$ with respect to 
this rule is defined by $\vartheta-\sum_{k, l} x_{k l}=\vartheta-\lim _{m, n \rightarrow \infty} \sum_{k, l=0}^{m, n} x_{k l}$. For short, throughout the text the summations without limits run from 0 to $\infty$, for example $\sum_{k, l} x_{k l}$ means that $\sum_{k, l=0}^{\infty} x_{k l}$.

The $\beta(\vartheta)$-dual $\lambda^{\beta(\vartheta)}$ with respect to the $\vartheta$-convergence of a double sequence space $\lambda$ is defined by

$$
\lambda^{\beta(\vartheta)}:=\left\{\left(a_{k l}\right) \in \Omega: \vartheta-\sum_{k, l} a_{k l} x_{k l} \text { exists for all }\left(x_{k l}\right) \in \lambda\right\}
$$

Let $A=\left(a_{m n k l}\right)$ be any four dimensional matrix. Then, a double sequence $x=\left(x_{k l}\right)$ is said to be $\vartheta$-summable with respect to $A$ if and only if

$$
(A x)_{m n}=\vartheta-\sum_{k, l} a_{m n k l} x_{k l}
$$

exists for each $m, n \in \mathbb{N}$. We define the $\vartheta$-summability domain $\lambda_{A}^{(\vartheta)}$ of $A$ in a double sequence space $\lambda$ by

$$
\lambda_{A}^{(\vartheta)}=\left\{x=\left(x_{k l}\right) \in \Omega: A x=\left(\vartheta-\sum_{k, l} a_{m n k l} x_{k l}\right)_{m, n \in \mathbb{N}} \text { exists and is in } \lambda\right\} .
$$

Here and after, unless stated otherwise we assume that $\vartheta$ denotes any of the symbols $p, b p$ or $r$. Let $\lambda$ and $\mu$ be two spaces of double sequences, and $A$ be a four dimensional matrix. Then, we say with the notation (2.1) that $A$ maps the space $\lambda$ into the space $\mu$ if $\lambda \subset \mu_{A}^{(\vartheta)}$ and we denote the set of all four dimensional matrices, transforming the space $\lambda$ into the space $\mu$, by $(\lambda: \mu)$. Thus, $A=\left(a_{m n k l}\right) \in(\lambda: \mu)$ if and only if the double series on the right side of (2.1) converges in the sense of $\vartheta$ for each $m, n \in \mathbb{N}$, i.e, $A_{m n} \in \lambda^{\beta(\vartheta)}$ for all $m, n \in \mathbb{N}$ and every $x \in \lambda$, and we have $A x \in \mu$ for all $x \in \lambda$; where $A_{m n}=\left(a_{m n k l}\right)_{k, l \in \mathbb{N}}$ for all $m, n \in \mathbb{N}$. Also by $(\lambda: \mu ; P)$, we denote the subclass of $(\lambda: \mu)$ of four dimensional matrices which preserve the limit or sum. We say that $A$ is $\mathfrak{C}_{\vartheta}$-conservative if $\mathcal{C}_{\vartheta} \subset\left(\mathcal{C}_{\vartheta}\right)_{A}$, and is $\mathcal{C}_{\vartheta}$-regular if it is $\mathcal{C}_{\vartheta}$-conservative and $\vartheta-\lim _{A} x=\vartheta-\lim _{m, n \rightarrow \infty}(A x)_{m n}=\vartheta-\lim _{m, n \rightarrow \infty} x_{m n}$, where $x=\left(x_{m n}\right) \in \mathcal{C}_{\vartheta}$.

Now, we may give the following two lemmas characterizing the classes $\left(\mathcal{C}_{b p}: \mathcal{C}_{b p} ; P\right)$ and $\left(\mathcal{M}_{u}: \mathcal{C}_{b p}\right)$ of four dimensional matrices. 
Lemma 2.1. [7, Theorem $2.2(\mathrm{~b})]$ Let $A=\left(a_{m n k l}\right)$ be any four dimensional matrix. Then, $A \in\left(\mathrm{C}_{b p}: \mathrm{C}_{b p} ; P\right)$ if and only if the following conditions hold:

$$
\begin{aligned}
& \sup _{m, n \in \mathbb{N}} \sum_{k, l}\left|a_{m n k l}\right|<\infty, \\
& b p-\lim _{m, n \rightarrow \infty} a_{m n k l}=0 \text { for all } k, l \in \mathbb{N}, \\
& b p-\lim _{m, n \rightarrow \infty} \sum_{k, l} a_{m n k l}=1, \\
& \text { there exists } l_{0} \in \mathbb{N} \text { such that } b p-\lim _{m, n \rightarrow \infty} \sum_{k}\left|a_{m n k l_{0}}\right|=0, \\
& \text { there exists } k_{0} \in \mathbb{N} \text { such that } b p-\lim _{m, n \rightarrow \infty} \sum_{l}\left|a_{m n k_{0} l}\right|=0 .
\end{aligned}
$$

Lemma 2.2. [8, Lemma 3.2] Let $A=\left(a_{m n k l}\right)$ be any four dimensional matrix. Then, $A \in\left(\mathcal{M}_{u}: \mathcal{C}_{b p}\right)$ if and only if the condition (2.2) holds and

$$
\begin{aligned}
& \text { there exists } a_{k l} \in \mathbb{C} \text { such that } b p-\lim _{m, n \rightarrow \infty} a_{m n k l}=a_{k l} \text { for all } k, l \in \mathbb{N}, \\
& b p-\lim _{m, n \rightarrow \infty} \sum_{k, l}\left|a_{m n k l}-a_{k l}\right|=0 \\
& b p-\lim _{m, n \rightarrow \infty} \sum_{l=0}^{n} a_{m n k l} \text { exists for each } k \in \mathbb{N}, \\
& b p-\lim _{m, n \rightarrow \infty} \sum_{k=0}^{m} a_{m n k l} \text { exists for each } l \in \mathbb{N} \\
& \sum_{k, l}\left|a_{m n k l}\right| \text { converges. }
\end{aligned}
$$

Definition 2.1. $[9,10]$ A four dimensional matrix $A$ is said to be $R H$-regular if it maps every bounded $p$-convergent double sequence into a $p$-convergent double sequence with the same $p$-limit.

Lemma 2.3. [11, Theorem 3.2] Let $A=\left(a_{m n k l}\right)$ be any four dimensional matrix. Then, $A \in\left(\mathcal{M}_{u}: \mathcal{M}_{u}\right)$ if and only if the condition (2.2) holds.

Mursaleen [12] introduced the concept of almost strong regularity for double sequences. A four dimensional matrix $A=\left(a_{m n k l}\right)$ is said to be almost strongly regular if for every $x=\left(x_{k l}\right) \in \mathcal{C}_{f}, A x \in \mathcal{C}_{f}$ with $f_{2}-\lim A x=f_{2}-\lim x$, i.e., it transforms every almost convergent double sequence into an almost convergent double sequence with the same limit. In the following lemma Mursaleen characterized the almost strongly regular matrices. 
Lemma 2.4. [12, Theorem 2.2] Let $A=\left(a_{m n k l}\right)$ be any four dimensional matrix. Then, $A \in\left(\mathcal{C}_{f}: \mathcal{C}_{f} ; P\right)$ if and only if condition (2.2) holds and

$$
\begin{aligned}
b p-\lim _{q, r \rightarrow \infty} \alpha(k, l, q, r, m, n) & =0 \text { uniformly in } m, n \in \mathbb{N} \text { for each } k, l \in \mathbb{N}, \\
b p-\lim _{q, r \rightarrow \infty} \sum_{k, l} \alpha(k, l, q, r, m, n) & =1 \text { uniformly in } m, n \in \mathbb{N},
\end{aligned}
$$$$
b p-\lim _{q, r \rightarrow \infty} \sum_{k}|\alpha(k, l, q, r, m, n)|=0 \text { uniformly in } m, n \in \mathbb{N} \text { for each } l \in \mathbb{N} \text {, }
$$$$
b p-\lim _{q, r \rightarrow \infty} \sum_{l}|\alpha(k, l, q, r, m, n)|=0 \text { uniformly in } m, n \in \mathbb{N} \text { for each } k \in \mathbb{N} \text {, }
$$

$b p-\lim _{q, r \rightarrow \infty} \sum_{k} \sum_{l}\left|\Delta_{10} \alpha(k, l, q, r, m, n)\right|=0$ uniformly in $m, n \in \mathbb{N}$,

$b p-\lim _{q, r \rightarrow \infty} \sum_{l} \sum_{k}\left|\Delta_{01} \alpha(k, l, q, r, m, n)\right|=0$ uniformly in $m, n \in \mathbb{N}$,

where

$$
\begin{aligned}
\alpha(k, l, q, r, m, n) & =\frac{1}{(q+1)(r+1)} \sum_{i=0}^{q} \sum_{j=0}^{r} a_{m+i, n+j, k l}, \\
\Delta_{10} \alpha(k, l, q, r, m, n) & =\alpha(k, l, q, r, m, n)-\alpha(k+1, l, q, r, m, n), \\
\Delta_{01} \alpha(k, l, q, r, m, n) & =\alpha(k, l, q, r, m, n)-\alpha(k, l+1, q, r, m, n) .
\end{aligned}
$$

Definition 2.2. If $A$ is a $\mathcal{C}_{\vartheta}$-conservative matrix, then we define the characteristic $\chi(A)$ of $A$ by

$$
\chi(A)=\vartheta-\lim _{m, n \rightarrow \infty} \sum_{k, l} a_{m n k l}-\sum_{k, l} \vartheta-\lim _{m, n \rightarrow \infty} a_{m n k l} .
$$

Definition 2.3. A $\mathcal{C}_{\vartheta}$-conservative matrix $A$ is called conull if $\chi(A)=0$ and is called coregular if $\chi(A) \neq 0$.

Remark 2.1. One can easily conclude by Lemma 2.1 and Lemma 2.4 that $\chi(A)=1$ whenever $A$ is a $\mathcal{C}_{b p}$-regular sequence to sequence four dimensional matrix or is an almost strongly regular four dimensional matrix. This leads to the fact that the class $\left(\mathcal{C}_{\vartheta}: \mathcal{C}_{\vartheta} ; P\right)$ of four dimensional matrices forms a subclass of the class of co-regular four dimensional matrices, where $\vartheta \in\{b p, f\}$.

Following Zeltser [13], we define the double sequences $\mathbf{e}^{\mathbf{k l}}=\left(e_{m n}^{k l}\right), \mathbf{e}^{\mathbf{l}}, \mathbf{e}_{\mathbf{k}}$, and $\mathbf{e}$ by

$$
\begin{aligned}
e_{m n}^{k l} & :=\left\{\begin{array}{rr}
1, & (k, l)=(m, n), \\
0, & \text { otherwise },
\end{array}\right. \\
\mathbf{e}^{\mathbf{l}} & :=\sum_{k} \mathbf{e}^{\mathbf{k} \mathbf{l},} \quad \mathbf{e}_{\mathbf{k}}:=\sum_{l} \mathbf{e}^{\mathbf{k l}}, \quad \text { and } \mathbf{e}:=\sum_{k, l} \mathbf{e}^{\mathbf{k l}}
\end{aligned}
$$

for all $k, l, m, n \in \mathbb{N}$. 
The original Steinhaus theorem was about the sequence to sequence matrix transformations which can be stated as "There is no matrix belonging to the classes of both Schur matrices and Toeplitz matrices". Later, the corresponding results were given by considering almost convergence instead of ordinary convergence, (cf. [2] and [18]). Moreover, Steinhaus type theorems for series to sequence and series to series matrix transformations were given by Başar and Solak [19].

In this study, our main goal is to give the corresponding Steinhaus type theorems for four dimensional matrix transformations. In order to achieve this, we characterize the classes $\left(\mathcal{M}_{u}: \mathcal{C}_{\vartheta}\right)$ with $\vartheta \in\{p, p 0, f\}$. However, since the characterization of the classes $\left(\mathcal{B S}: \mathcal{C}_{f}\right),\left(\mathcal{B S}: \mathcal{C S}_{\vartheta}\right),\left(\mathcal{C S}_{\vartheta}: \mathcal{C}_{\vartheta} ; P\right)$ and $\left(\mathcal{C S}_{\vartheta}: \mathcal{C S}_{\vartheta} ; P\right)$ with $\vartheta \in\{p, p 0, b p, r, f\}$ are still open, we are not able to present Steinhaus type theorems for series to sequence and series to series four dimensional matrix transformations.

\section{MAin Results}

In this section, we characterize the classes $\left(\mathcal{M}_{u}: \mathcal{C}_{\vartheta}\right)$ and we give two Steinhaus type theorems, where $\vartheta \in\{p, p 0, f\}$.

Following Maddox [14], we formulate the Steinhaus type theorems for four dimensional matrix transformations, as follows:

Definition 3.1. Let $X, y$ and $z$ be given double sequence spaces such that $z \supset X$ and suppose that there is some notion of limit or sum in the spaces $\mathcal{X}$ and $\mathcal{Y}$. Then, we say the result of the form $(x: y ; P) \cap(z: y)=\emptyset$ as a Steinhaus type theorem, where $\emptyset$ denotes the empty set.

Using convergence in the Pringsheim's sense, Patterson [15] gave the multidimensional analogue of Steinhaus's theorem whose original proof can be found in Cooke [16, pp. 75-76], as follows:

Theorem 3.1. [15, Theorem 3.1] If $A$ is an RH-regular matrix, then there exists a bounded double sequence $x$ consisting only of 0 's and 1 's which is not A-summable.

Now, we can give our theorem about the multidimensional analogue of Steinhaus's theorem.

Theorem 3.2. The classes $\left(\mathcal{C}_{b p}: \mathcal{C}_{b p} ; P\right)$ and $\left(\mathcal{M}_{u}: \mathfrak{C}_{b p}\right)$ are disjoint.

Proof. Suppose that the classes $\left(\mathcal{C}_{b p}: \mathcal{C}_{b p} ; P\right)$ and $\left(\mathcal{M}_{u}: \mathcal{C}_{b p}\right)$ are not disjoint. Then, there exists at least one four dimensional matrix $A$ such that $A \in\left(\mathcal{C}_{b p}: \mathcal{C}_{b p} ; P\right) \cap\left(\mathcal{M}_{u}\right.$ : $\left.\mathfrak{C}_{b p}\right)$. Since $A \in\left(\mathcal{C}_{b p}: \mathcal{C}_{b p} ; P\right), \chi(A)=1$ by Lemma 2.1. Since $A \in\left(\mathcal{M}_{u}: \mathcal{C}_{b p}\right)$, combining the relations $(2.3)$ and $(2.4)$ we have $\chi(A)=0$, a contradiction. That is to say that the classes $\left(\mathcal{C}_{b p}: \mathcal{C}_{b p} ; P\right)$ and $\left(\mathcal{M}_{u}: \mathcal{C}_{b p}\right)$ of four dimensional matrices are disjoint, as asserted. 
Theorem 3.3. Let $A=\left(a_{m n k l}\right)$ be any four dimensional matrix. Then, $A \in\left(\mathcal{M}_{u}: \mathcal{C}_{p 0}\right)$ if and only if

$$
p-\lim _{m, n \rightarrow \infty} a_{m n k l}=0 \text { for each } k, l \in \mathbb{N},
$$

(3.2) for every $l \in \mathbb{N}$, there exists $K \in \mathbb{N}$ such that $a_{m n k l}=0$ for all $m, n, k>K$,

(3.3) for every $k \in \mathbb{N}$, there exists $L \in \mathbb{N}$ such that $a_{m n k l}=0$ for all $m, n, l>L$.

Proof. Suppose that the conditions (3.1)-(3.3) hold, and let $x=\left(x_{k l}\right) \in \mathcal{M}_{u}$. Let $n_{0}=\max \{L, K\} \in \mathbb{N}$. Hence, for every $\varepsilon>0$ there exists $n_{0}=n_{0}(\varepsilon) \in \mathbb{N}$ such that

$$
\begin{aligned}
\left|(A x)_{m n}\right| & =\left|\sum_{k, l} a_{m n k l} x_{k l}\right| \leq\|x\|_{\infty} \sum_{k, l}\left|a_{m n k l}\right| \\
& =\|x\|_{\infty}\left[\sum_{k, l=0}^{n_{0}}\left|a_{m n k l}\right|+\sum_{k=0}^{n_{0}} \sum_{l=n_{0}+1}^{\infty}\left|a_{m n k l}\right|+\sum_{k=n_{0}+1}^{\infty} \sum_{l=0}^{n_{0}}\left|a_{m n k l}\right|+\sum_{k, l=n_{0}}^{\infty}\left|a_{m n k l}\right|\right] \\
& <\varepsilon\|x\|_{\infty}\left(n_{0}+1\right)^{2},
\end{aligned}
$$

for all $m, n>n_{0}$, that is, $A x \in \mathcal{C}_{p 0}$.

Conversely, suppose that $A=\left(a_{m n k l}\right) \in\left(\mathcal{M}_{u}: \mathcal{C}_{p 0}\right)$. Then, $A x$ exists and belongs to $\mathcal{C}_{p 0}$ for all $x \in \mathcal{M}_{u}$. If we take $x=\mathbf{e}^{\mathrm{kl}}$ for each $k, l \in \mathbb{N}, x=\mathbf{e}^{\mathbf{l}}, x=\mathbf{e}_{\mathbf{k}}$ and $x=\mathbf{e}$ defined by (2.7), respectively, then we have

$$
\begin{aligned}
& 0=p-\lim _{m, n \rightarrow \infty}\left(A \mathbf{e}^{\mathbf{k} \mathbf{l}}\right)_{m n}=p-\lim _{m, n \rightarrow \infty} a_{m n k l} \text { for each } k, l \in \mathbb{N}, \\
& 0=p-\lim _{m, n \rightarrow \infty}\left(A \mathbf{e}_{\mathbf{k}}\right)_{m n}=p-\lim _{m, n \rightarrow \infty} \sum_{l} a_{m n k l} \text { for each } k \in \mathbb{N}, \\
& 0=p-\lim _{m, n \rightarrow \infty}\left(A \mathbf{e}^{\mathbf{l}}\right)_{m n}=p-\lim _{m, n \rightarrow \infty} \sum_{k} a_{m n k l} \text { for each } l \in \mathbb{N}, \\
& 0=p-\lim _{m, n \rightarrow \infty}(A \mathbf{e})_{m n}=p-\lim _{m, n \rightarrow \infty} \sum_{k, l} a_{m n k l} .
\end{aligned}
$$

In order to the relation (3.4) to be valid, there must exist a $K \in \mathbb{N}$ such that $a_{m n k l}=0$ for all $m, n, k>K$, where $K$ may depend on $l$. Otherwise there exist non-decreasing unbounded three sequences of positive integers $\left(m_{j}\right),\left(n_{j}\right)$ and $\left(k_{j}\right)$ for $j \in \mathbb{N}$ such that $a_{m_{j}, n_{j}, k_{j}, l} \neq 0$ for each $l \in \mathbb{N}$. If we take $x=\mathbf{e}^{\mathbf{l}}$ and

$$
\left|a_{m_{j} n_{j} k_{j} l}\right|>\left|\sum_{i=0}^{j-1} a_{m_{j} n_{j} k_{i} l}\right|+K
$$

then we have

$$
\left|\sum_{i=0}^{j} a_{m_{j} n_{j} k_{i} l}\right| \geq\left|a_{m_{j} n_{j} k_{j} l}\right|-\left|\sum_{i=0}^{j-1} a_{m_{j} n_{j} k_{i} l}\right|>K,
$$


that is, $\left|\sum_{i=0}^{j} a_{m_{j} n_{j} k_{i} l}\right| \rightarrow \infty$, as $j \rightarrow \infty$. Using the relation

$$
\left(A \mathbf{e}^{\mathrm{l}}\right)_{m_{j} n_{j}}=\sum_{k} a_{m_{j} n_{j} k l}=\lim _{r \rightarrow \infty} \sum_{k=0}^{r} a_{m_{j} n_{j} k l},
$$

we can say that $(A x)_{m n} \nrightarrow 0$, as $m, n \rightarrow \infty$, a contradiction. That is to say that the condition (3.2) is necessary. Similarly, we obtain the necessity of the condition (3.3). Also, one can see by taking $x=\mathbf{e}$ that the relation (3.5) is the consequence of (3.2) and (3.3). This step completes the proof.

Theorem 3.4. Let $A=\left(a_{m n k l}\right)$ be any four dimensional matrix. Then, $A \in\left(\mathcal{M}_{u}: \mathcal{C}_{p}\right)$ if and only if the conditions (3.2)-(3.3) hold, and

$$
\text { there exists } \alpha_{k l} \in \mathbb{C} \text { such that } p-\lim _{m, n \rightarrow \infty} a_{m n k l}=\alpha_{k l} \text {. }
$$

Proof. Suppose that $A=\left(a_{m n k l}\right) \in\left(\mathcal{M}_{u}: \mathcal{C}_{p}\right)$. Then, $A x$ exists and belongs to $\mathcal{C}_{p}$ for any $x \in \mathcal{M}_{u}$. We write

$$
\begin{aligned}
(A x)_{m+r, n+s}-(A x)_{m n} & =\sum_{k, l} a_{m+r, n+s, k l} x_{k l}-\sum_{k, l} a_{m n k l} x_{k l} \\
& =\sum_{k, l}\left(a_{m+r, n+s, k l}-a_{m n k l}\right) x_{k l} \\
& =(B x)_{m n},
\end{aligned}
$$

for all $m, n \in \mathbb{N}$, where the matrix $B=\left(b_{m n k l}^{r s}\right)$ is defined by $b_{m n k l}^{r s}:=a_{m+r, n+s, k l}-a_{m n k l}$ for all $m, n, r, s, k, l \in \mathbb{N}$. By letting $p$-limit in the relation (3.7) as $m, n \rightarrow \infty$, we obtain that $p-\lim _{m, n \rightarrow \infty}(B x)_{m n}=0$. Hence, $B \in\left(\mathcal{M}_{u}: \mathcal{C}_{p 0}\right)$. From the condition (3.1), $p-\lim _{m, n \rightarrow \infty} a_{m n k l}$ exists for each $k, l \in \mathbb{N}$, say $\alpha_{k l}$ which is in $\mathbb{C}$. So, the condition (3.6) is necessary. Following the similar way used in the proof of Theorem 3.3, one can establish the necessity of the conditions (3.2) and (3.3).

Conversely, suppose that the conditions (3.2), (3.3) and (3.6) hold, and let $x=$ $\left(x_{k l}\right) \in \mathcal{M}_{u}$. Let $n_{0}=\max \{L, K\} \in \mathbb{N}$. Hence, for every $\varepsilon>0$ there exists $n_{0}=$ $n_{0}(\varepsilon) \in \mathbb{N}$ such that

$$
\begin{aligned}
\left|\sum_{k, l} a_{m n k l} x_{k l}-\sum_{k, l=0}^{n_{0}} \alpha_{k l} x_{k l}\right| & =\left|\sum_{k, l=0}^{n_{0}} a_{m n k l} x_{k l}-\sum_{k, l=0}^{n_{0}} \alpha_{k l} x_{k l}\right| \\
& =\left|\sum_{k, l=0}^{n_{0}}\left(a_{m n k l}-\alpha_{k l}\right) x_{k l}\right| \\
& \leq\|x\|_{\infty} \sum_{k, l=0}^{n_{0}}\left|a_{m n k l}-\alpha_{k l}\right| \\
& <\varepsilon\|x\|_{\infty}\left(n_{0}+1\right)^{2},
\end{aligned}
$$

for all $m, n>n_{0}$, that is, $p-\lim _{m, n \rightarrow \infty}(A x)_{m n}=\sum_{k, l=0}^{n_{0}} \alpha_{k l} x_{k l}$, as desired. 
Theorem 3.5. Let $A=\left(a_{m n k l}\right)$ be any four dimensional matrix. Then, $A \in\left(\mathcal{M}_{u}: \mathcal{C}_{f}\right)$ if and only if the condition (2.2) holds and there exists $a_{k l} \in \mathbb{C}$ such that

(3.8) $f_{2}-\lim _{m, n \rightarrow \infty} a_{m n k l}=a_{k l}$ for all $k, l \in \mathbb{N}$,

(3.9) for every $m, n, l \in \mathbb{N}$,

there exists $K \in \mathbb{N} \ni \frac{1}{(q+1)(r+1)} \sum_{i=0}^{q} \sum_{j=0}^{r} a_{m+i, n+j, k l}=0$ for all $q, r, k>K$,

for every $m, n, k \in \mathbb{N}$,

there exists $L \in \mathbb{N} \ni \frac{1}{(q+1)(r+1)} \sum_{i=0}^{q} \sum_{j=0}^{r} a_{m+i, n+j, k l}=0$ for all $q, r, l>L$.

Proof. Suppose that $A=\left(a_{m n k l}\right) \in\left(\mathcal{M}_{u}: \mathcal{C}_{f}\right)$. Then, $A x$ exists and belongs to $\mathcal{C}_{f}$ for all $x \in \mathcal{M}_{u}$. Also, it is known by [17] that the inclusion $\mathcal{C}_{f} \subset \mathcal{M}_{u}$ holds. So, the inclusion $\left(\mathcal{M}_{u}: \mathcal{C}_{f}\right) \subset\left(\mathcal{M}_{u}: \mathcal{M}_{u}\right)$ holds which gives the necessity of the condition (2.2) by Lemma 2.3. Since $A x$ belongs to $\mathcal{C}_{f}$ for all $x \in \mathcal{M}_{u}$, if we take $x=\mathrm{e}^{\mathrm{kl}}$ for each $k, l \in \mathbb{N}$, then we obtain the necessity of the conditions (3.8). If we consider the sequences $x=\mathbf{e}^{\mathbf{l}}, x=\mathbf{e}_{\mathbf{k}}$ and $x=\mathbf{e}$, then we derive the conditions

$$
\begin{aligned}
& f_{2}-\lim _{m, n \rightarrow \infty} \sum_{l} a_{m n k l} \text { exists for each } k \in \mathbb{N}, \\
& f_{2}-\lim _{m, n \rightarrow \infty} \sum_{k} a_{m n k l} \text { exists for each } l \in \mathbb{N}, \\
& f_{2}-\lim _{m, n \rightarrow \infty} \sum_{k, l} a_{m n k l} \text { exists. }
\end{aligned}
$$

Define the double sequence $\left(B_{m n}\right)$ of four dimensional matrices for all $m, n, q, r, k, l \in$ $\mathbb{N}$, as follows:

$$
b_{q r k l}^{m n}:=\frac{1}{(q+1)(r+1)} \sum_{i=0}^{q} \sum_{j=0}^{r} a_{m+i, n+j, k l} .
$$

Therefore, we obtain for $x=\left(x_{k l}\right) \in \mathcal{M}_{u}$ that

$$
\begin{aligned}
\left(B_{m n} x\right)_{q r} & =\sum_{k, l} b_{q r k l}^{m n} x_{k l} \\
& =\frac{1}{(q+1)(r+1)} \sum_{i=0}^{q} \sum_{j=0}^{r} \sum_{k, l} a_{m+i, n+j, k l} x_{k l} \\
& =\frac{1}{(q+1)(r+1)} \sum_{i=0}^{q} \sum_{j=0}^{r}(A x)_{m+i, n+j} .
\end{aligned}
$$


Then, since $A x$ belongs to $\mathcal{C}_{f}$ for all $x \in \mathcal{M}_{u}, f_{2}-\lim A x$ exists, that is,

$$
p-\lim _{q, r \rightarrow \infty} \frac{1}{(q+1)(r+1)} \sum_{i=0}^{q} \sum_{j=0}^{r}(A x)_{m+i, n+j}
$$

exists, uniformly in $m$ and $n$. From the relations (3.15) and (3.16), we conclude that $B_{m n} x$ exists for all $x \in \mathcal{M}_{u}$, that is, $B_{m n} \in\left(\mathcal{M}_{u}: \mathcal{C}_{p}\right)$. So, by Theorem 3.4 we have

there exists $a_{k l} \in \mathbb{C}$ such that $p-\lim _{q, r \rightarrow \infty} b_{q r k l}^{m n}=a_{k l}$ for all $m, n, k, l \in \mathbb{N}$,

for every $m, n, l \in \mathbb{N}$, there exists $K \in \mathbb{N}$ such that $b_{q r k l}^{m n}=0$ for all $q, r, k>K$,

for every $m, n, k \in \mathbb{N}$, there exists $L \in \mathbb{N}$ such that $b_{q r k l}^{m n}=0$ for all $q, r, l>L$.

The relation (3.17) is equivalent to the condition (3.8). Also, the conditions (3.8) and (3.18) include the condition (3.11). Similarly, the conditions (3.8) and (3.19) include the condition (3.12). Finally, the conditions (3.8), (3.18) and (3.19) include the condition (3.13).

The sufficiency of the theorem can be proved by the similar way used in the proof of Theorem 3.3. To avoid the repetition of the similar statements, we omit the detail.

Theorem 3.6. The classes $\left(\mathcal{M}_{u}: \mathcal{C}_{f}\right)$ and $\left(\mathcal{C}_{f}: \mathcal{C}_{f} ; P\right)$ are disjoint.

Proof. Suppose that $\left(\mathcal{M}_{u}: \mathcal{C}_{f}\right) \cap\left(\mathcal{C}_{f}: \mathcal{C}_{f} ; P\right) \neq \emptyset$. Then, there exists at least one four dimensional matrix $A$ such that $A \in\left(\mathcal{M}_{u}: \mathcal{C}_{f}\right) \cap\left(\mathcal{C}_{f}: \mathcal{C}_{f} ; P\right)$. Since $A=\left(a_{m n k l}\right) \in\left(\mathcal{C}_{f}: \mathcal{C}_{f} ; P\right)$, the condition (2.5) holds, i.e.

$$
b p-\lim _{q, r \rightarrow \infty} \frac{1}{(q+1)(r+1)} \sum_{i=0}^{q} \sum_{j=0}^{r} a_{m+i, n+j, k l}=0 \text { uniformly in } m, n \in \mathbb{N} .
$$

Hence, $b p-\lim _{q, r \rightarrow \infty} b_{q r k l}^{m n}=0$, where $b_{q r k l}^{m n}$ is given by (3.14). Let $n_{0}=\max \{K, L\} \in \mathbb{N}$. Since $A \in\left(\mathcal{M}_{u}: \mathcal{C}_{f}\right)$, the conditions (3.9) and (3.10) hold. So, for every $m, n \in \mathbb{N}$, there exists $n_{0} \in \mathbb{N}$ such that $b_{q r k l}^{m n}=0$ for all $q, r, k, l>n_{0}$. Hence,

$$
b p-\lim _{q, r \rightarrow \infty} \sum_{k, l} b_{q r k l}^{m n}=b p-\lim _{q, r \rightarrow \infty} \sum_{k, l=0}^{n_{0}} b_{q r k l}^{m n}=0
$$

uniformly in $m, n \in \mathbb{N}$ which contradicts the condition (2.6).

Acknowledgements. The authors are indebted to the referee for helpful suggestions and insights concerning the presentation of this paper. 


\section{REFERENCES}

[1] C. Eizen and G. Laush, Infinite matrices and almost convergence, Math. Japon 14 (1969), 137143.

[2] F. Başar, f-conservative matrix sequences, Tamkang J. Math. 22 (2) (1991), 205-212.

[3] A. Pringsheim, Zur theorie der zweifach unendlichen Zahlenfolgen, Math. Ann. 53 (1900), 289321.

[4] F. Móricz, Extensions of the spaces $c$ and $c_{0}$ from single to double sequences, Acta Math. Hungar. 57 (1991), 129-136.

[5] F. Móricz and B. E. Rhoades, Almost convergence of double sequences and strong regularity of summability matrices, Math. Proc. Camb. Phil. Soc. 104 (1988), 283-294.

[6] G. G. Lorentz, A contribution to the theory of divergent sequences, Acta Math. 80 (1948), 167-190.

[7] M. Zeltser, M. Mursaleen and S. A. Mohiuddine, On almost conservative matrix methods for double sequence spaces, Publ. Math. Debrecen 75 (2009), 387-399.

[8] C. Çakan, B. Altay and M. Mursaleen, The $\sigma$-convergence and $\sigma$-core of double sequences, Appl. Math. Letters, 19 (2006), 1122-1128.

[9] H. J. Hamilton, Transformations of multiple sequences, Duke Math. J. 2 (1936), 29-60.

[10] G. M. Robison, Divergent double sequences and series, Trans. Am. Math. Soc. 28 (1926), 50-73.

[11] M. Yeşilkayagil and F. Başar, Mercerian theorem for four dimensional matrices, Commun. Fac. Sci. Univ. Ank. Ser. A 65 (2) (2016), to appear.

[12] M. Mursaleen, Almost strongly regular matrices and a core theorem for double sequences, J. Math. Anal. Appl. 293 (2) (2004), 523-531.

[13] M. Zeltser, On conservative matrix methods for double sequence spaces, Acta Math. Hung. 95 (3) (2002), 225-242.

[14] I. J. Maddox, On theorems of Steinhaus type, J. London Math. Soc. 42 (1967), 239-244.

[15] R. F. Patterson, Analogues of some fundamental theorems of summability theory, Int. J. Math. Math. Sci. 23 (1) (2000), 1-9.

[16] R. C. Cooke, Infinite Matrices and Sequence Spaces, Macmillan and Co. Limited, London, 1950.

[17] M. Mursaleen and S. A. Mohiuddine, Convergence Methods For Double Sequences and Applications, Springer, Heidelberg, 2014.

[18] F. Başar, Summability Theory and Its Applications, Bentham Science Publishers, İstanbul, 2012.

[19] F. Başar and İ. Solak, Almost-coercive matrix transformations, Rend. Mat. Appl. 11 (2) (1991), 249-256.

${ }^{1}$ Department of Mathematics,

UŞAK UNIVERSITY,

1 EYlül CAMPus, 64200 - UşaK, TURKEY

E-mail address: medine.yesilkayagil@usak.edu.tr

${ }^{2}$ Department of Mathematics,

FATIH UNIVERSITY,

The Hadimköy CAmpus, BüYÜKÇEKMECE, 34500 - İstanbul, TurkeY

E-mail address: fbasar@fatih.edu.tr, feyzibasar@gmail.com 